Article

\title{
Simultaneous Determination of Four Tanshinones by UPLC-TQ/MS and Their Pharmacokinetic Application after Administration of Single Ethanol Extract of Danshen Combined with Water Extract in Normal and Adenine-Induced Chronic Renal Failure Rats
}

\author{
Hong-Die Cai ${ }^{1}$, Shu-Lan Su ${ }^{1, *}$, Yonghui Li ${ }^{2}$, Zhenhua Zhu ${ }^{1}$, Jianming Guo ${ }^{1}$, Yue Zhu ${ }^{1}$, \\ Sheng Guo ${ }^{1}$, Dawei Qian ${ }^{1}$ and Jinao Duan ${ }^{1, *}$ \\ 1 Jiangsu Collaborative Innovation Center of Chinese Medicinal Resources Industrialization, \\ National and Local Collaborative Engineering Center of Chinese Medicinal Resources Industrialization and \\ Formulae Innovative Medicine, and Jiangsu Key Laboratory for High Technology Research of TCM \\ Formulae, Nanjing University of Chinese Medicine, Nanjing 210023, China; \\ caihongdie@foxmail.com (H.-D.C.); 04040416@163.com (Z.Z.); guojianming@163.com (J.G.); \\ zhuyue@163.com (Y.Z.); gsh916@163.com (S.G.); qiandwnj@126.com (D.Q.) \\ 2 Hainan Provincial Key Laboratory of R\&D of Tropical Herbs, School of Pharmacy, \\ Hainan Medical University, Haikou 571199, China; lyhssl@126.com \\ * Correspondence: sushulan1974@njucm.edu.cn (S.-L.S.); duanja@163.com (J.D.); \\ Tel./Fax: +86-25-8581-1917 (S.-L.S.); +86-25-8581-1116 (J.D.)
}

Academic Editor: Derek J. McPhee

Received: 19 October 2016; Accepted: 25 November 2016; Published: 28 November 2016

\begin{abstract}
Salvia miltiorrhiza, one of the major traditional Chinese medicines, is commonly used and the main active ingredients-tanshinones-possess the ability to improve renal function. In this paper, the UPLC-TQ/MS method of simultaneously determining four tanshinones-tanshinone IIA, dihydrotanshinone I, tanshinone I, and cryptotanshinone-was established and applied to assess the pharmacokinetics in normal and chronic renal failure (CRF) rat plasma. The pharmacokinetics of tanshinones in rats were studied after separately intragastric administration of Salvia miltiorrhiza ethanol extract (SMEE) $(0.65 \mathrm{~g} / \mathrm{kg})$, SMEE $(0.65 \mathrm{~g} / \mathrm{kg})$ combined with Salvia miltiorrhiza water extract (SMWE) $(1.55 \mathrm{~g} / \mathrm{kg})$. The results showed $\mathrm{C}_{\max }$ and $\mathrm{AUC}_{0-\mathrm{t}}$ of tanshinone IIA, tanshinone I, cryptotanshinone reduced by $50 \% \sim 80 \%$ and CLz/F increased by $2 \sim 4$ times $(p<0.05)$ in model group after administrated with SMEE. Nevertheless, after intragastric administration of a combination of SMWE and SMEE, the $\mathrm{C}_{\max }$ and $\mathrm{AUC}_{0-\mathrm{t}}$ of four tanshinones were upregulated and CLz/F was downregulated, which undulated similarity from the model group to the normal group with compatibility of SMEE and SMWE. These results hinted that SMWE could improve the bioavailability of tanshinones in CRF rats, which provides scientific information for further exploration the mechanism of the combination of SMWE and SMEE and offers a reference for clinical administration of Salvia miltiorrhiza.
\end{abstract}

Keywords: Salvia miltiorrhiza Bge.; tanshinones; pharmacokinetics; UPLC-TQ/MS; chronic renal failure

\section{Introduction}

Salvia miltiorrhiza, a traditional Chinese herbal medicine, derived from the root of Salvia miltiorrhiza and belonging to the Salvia genus of family of Labiatae, has been used widely in clinics for the treatment of coronary heart diseases, particularly angina pectoris and myocardial infarction $[1,2]$. 
Previous research demonstrated that Salvia miltiorrhiza possessed various pharmacological effects for improving cerebral ischemia reperfusion injury, blood rheology, platelet function, anti-hypertensive, anti-inflammatory, and protecting the cardiovascular system [3-7].

Active ingredients in Salvia miltiorrhiza mainly include hydrosoluble salvianolic acids and liposoluble tanshinones. Pharmacological activities of salvianolic acids include anticancer activity on head and neck cancer and precancer cells [8], protection against polychlorinated biphenyls-induced oxidative stress [9], amelioration of central nervous system autoimmunity by suppressing Th1 responses [10], improvement of TNF- $\alpha$ induced cerebral microcirculatory changes in a micro-invasive mouse model [11], inhibition of endothelial dysfunction and vascular remodeling in spontaneously hypertensive rats [12], and so on. Tanshinones process anti-inflammatory activities in THP-1 macrophages [13], inhibit human esophageal cancer cell growth [14], suppress disruption of the blood-brain barrier [15], ameliorate bleomycin-induced pulmonary fibrosis [16], and prevent the loss of nigrostriatal dopaminergic neurons [17], and so on. Moreover, research shows Salvia miltiorrhiza extract, containing salvianolic acids and tanshinones, can prevent renal interstitial fibrosis in rats by regulating the expression of TSP-1, TGF- $\beta 1$, VEGF [18]. Salvianolate can improve renal function and alleviate the progress of CRF by increasing renal blood flow, decreasing the renal oxygen consumption and improving energy metabolism in renal tissues [19]. Salvianolic acid B exhibited protection on renal interstitial fibrosis in rats induced by $\mathrm{HgCl}_{2}$ by increasing organism function against oxidative damage and alleviating lipid oxidation injury [20]. Tanshinone IIA significantly attenuated renal fibrosis in five out of six nephrectomized rats [21] and retarded glomerular sclerosis by enhancing the expression of nephrin and inhibiting the expression of TGF- $\beta 1$ in kidney [22]. The large aggregation of studies demonstrated the pharmacokinetic profile of tanshinone IIA, cryptotanshinone, dihydrotanshinone $\mathrm{I}$, and tanshinone I in normal rats with intragastric administration [23-25]. However, the biological environment in the body in a pathological state was different from its normal status, such as the variation of intestinal microecology in CRF Patients [26]. The pharmacokinetic profile after intragastric administration was closely associated with intestinal absorption. Therefore, it is necessary to explore the pharmacokinetic profile of tanshinones in CRF rats. Furthermore, studies showed the effective constituents in liposoluble or water extract of Salvia miltiorrhiza can interact in normal rats, changing pharmacokinetic parameters of various constituents [27-30]. Hence, the pharmacokinetic process of any single component cannot accurately represent the pharmacokinetic process of Salvia miltiorrhiza. Furthermore, the influence of water-soluble compounds on the pharmacokinetic profile of tanshinones in CRF rats was seldom reported. Therefore, multi-component pharmacokinetic research of SM would provide a reference for clinical applications in CRF.

The aim of the study is to establish a new UPLC-TQ/MS method based on the previous study method [31] and to explore and compare the difference of the pharmacokinetic profiles of tanshinones in normal and CRF rats and evaluate the influence of water extract of Salvia miltiorrhiza on the pharmacokinetic parameters of tanshinones in normal and CRF rats, providing a reference for clinical administration of Salvia miltiorrhiza.

\section{Results and Discussion}

\subsection{Components Analysis of SMEE and SMWE}

The constituents in SMEE were mainly tanshinones, including tanshinone IIA, cryptotanshinone, dihydrotanshinone I, tanshinone I, with the contents of $12.63 \mathrm{mg} / \mathrm{g}, 43.73 \mathrm{mg} / \mathrm{g}, 10.27 \mathrm{mg} / \mathrm{g}$, and $6.25 \mathrm{mg} / \mathrm{g}$, respectively. While the constituents in SMWE were water-soluble compounds of salvianoli acids including danshensu at $0.51 \mathrm{mg} / \mathrm{g}$, rosmarinic acid at $3.76 \mathrm{mg} / \mathrm{g}$, lithospermic acid at $1.94 \mathrm{mg} / \mathrm{g}$, salvianoli acid A at $1.71 \mathrm{mg} / \mathrm{g}$, and salvianoli acid B at $85.47 \mathrm{mg} / \mathrm{g}$. The contents of these compounds were determined by UPLC-TQ/MS. The RSD of stability and repeatability was below $10 \%$. 


\subsection{Optimization of Mass Spectrometry Conditions}

To obtain the best mass spectrometry conditions, the standard solutions of the analytes and internal standard (IS) were separately infused into the mass spectrometer, and were studied in both positive and negative ion modes. The results showed the four analytes and IS had a stronger response in positive ion mode. Product ions were automatically chosen according to the stability and ion response by MS. Declustering potential (DP) and collision energy (CE) for each analyte and IS were also automatically optimized. The optimized MS/MS transitions and MS parameters of four analytes and IS are summarized in Table 1.

Table 1. The retention time $\left(t_{R}\right)$, optimized MS/MS transitions, declustering potential (DP) and collision energy (CE) for four analytes and IS.

\begin{tabular}{cccccc}
\hline Analytes & $\mathbf{t}_{\mathbf{R}}(\mathbf{m i n})$ & Precursor Ion $(\mathbf{m} / \mathbf{z})$ & Product Ion $(\mathbf{m} / \mathbf{z})$ & DP $(\mathbf{V})$ & $\mathbf{C E}(\mathbf{e V})$ \\
\hline Tanshinone IIA & 10.87 & 295.1 & 190.8 & 32 & 44 \\
Dihydrotanshinone I & 9.80 & 279.2 & 189.9 & 28 & 34 \\
Cryptotanshinone & 10.34 & 297.1 & 251.1 & 36 & 26 \\
Tanshinone I & 10.36 & 277.2 & 178.0 & 30 & 36 \\
Clarithromycin (IS) & 7.56 & 748.6 & 82.9 & 24 & 46 \\
\hline
\end{tabular}

\subsection{Method Validation}

\subsubsection{Specificity}

Mixed blank plasma samples from six rats were used to investigate the specificity of the method. Representative multiple-reaction monitoring chromatograms of blank plasma, blank plasma spiked with middle concentration standard solution and IS, as well as plasma samples at $2 \mathrm{~h}$ after oral administration of SMEE to rats spiked with IS are shown in Figure 1. No endogenous interference from the plasma was observed.
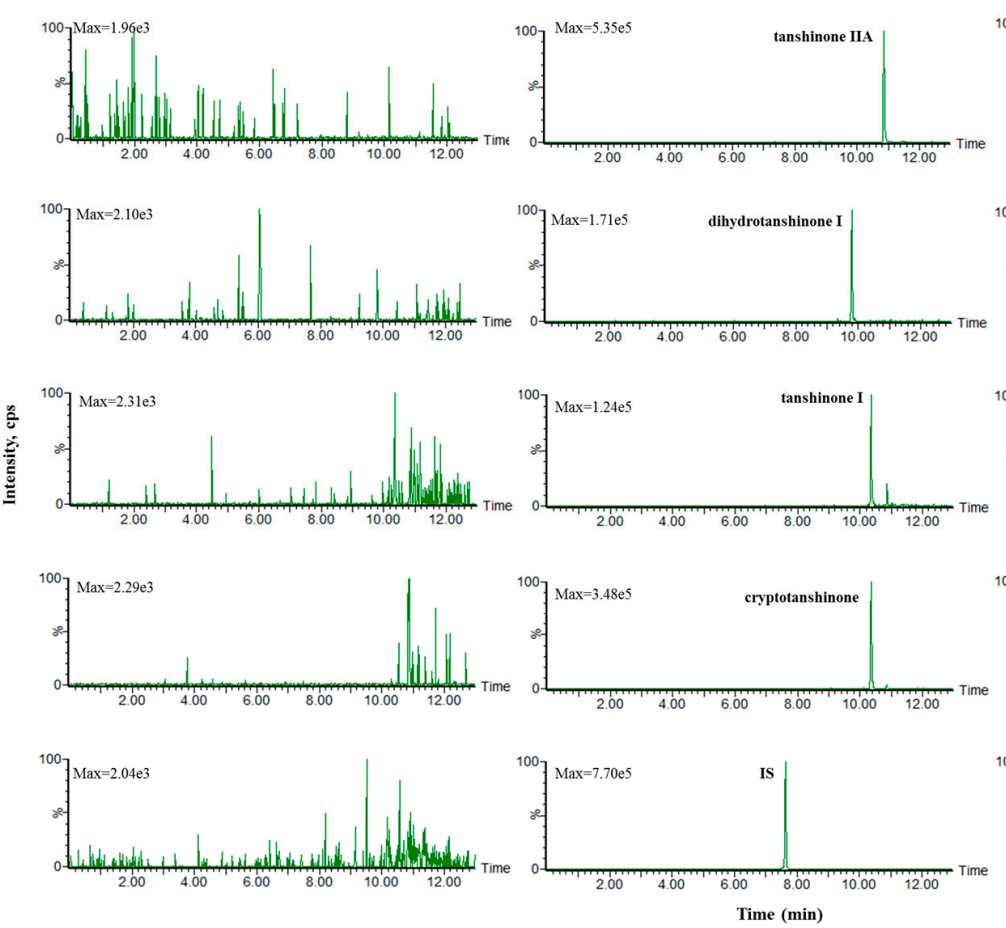
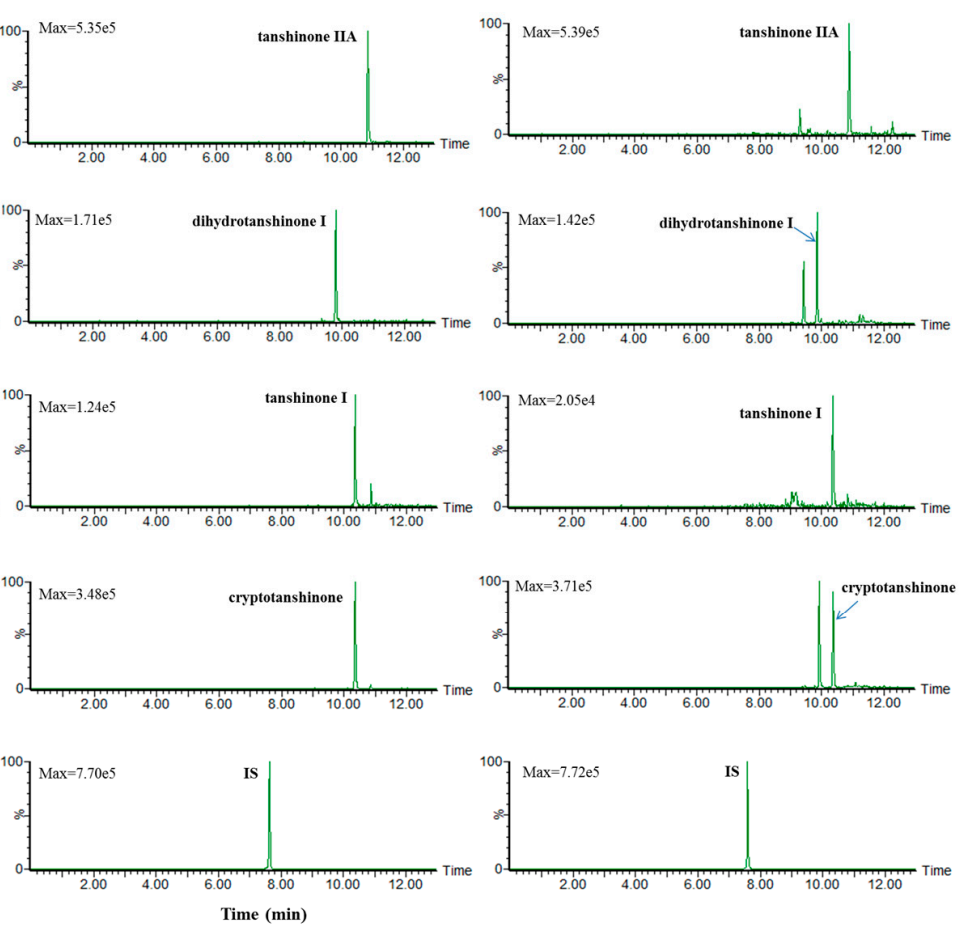

Figure 1. Representative multiple-reaction monitoring chromatograms of (A) blank plasma; (B) the blank plasma spiked with middle concentration standard solution and IS; (C) plasma samples at $2 \mathrm{~h}$ after oral administration of SMEE to rats spiked with IS. 


\subsubsection{Linearity of Calibration Curves and LLOQs}

The calibration curves of four tanshinones exhibited good linearity with correlation coefficients $\left(\mathrm{R}^{2}\right)$ within the range of 0.991-0.999. The calibration curves and LLOQs of the four tanshinones are summarized in Table 2.

Table 2. The regression equations, linear ranges, correlation coefficient $\left(R^{2}\right)$, and LLOQs for four tanshinones in rat plasma $(n=6)$.

\begin{tabular}{cccccc}
\hline Analytes & Regression Equation & $\mathbf{R}^{2}$ & $\begin{array}{c}\text { Linearity } \\
\text { Range }(\mathbf{n g} / \mathbf{m L})\end{array}$ & $\begin{array}{c}\text { LLOQ } \\
(\mathbf{n g} / \mathbf{m L})\end{array}$ & $\begin{array}{c}\text { CV \%o of } \\
\text { Calibration Slopes }\end{array}$ \\
\hline Tanshinone IIA & $y=0.0195 x+0.0258$ & 0.9990 & $0.540-108$ & 0.540 & 0.500 \\
Dihydrotanshinone I & $y=0.0119 x+0.0073$ & 0.9993 & $0.570-114$ & 0.570 & 0.350 \\
Cryptotanshinone & $y=0.0224 x+0.0317$ & 0.9993 & $0.555-111$ & 0.555 & 0.350 \\
Tanshinone I & $y=0.0107 x+0.0305$ & 0.9974 & $0.535-107$ & 0.535 & 1.301 \\
\hline
\end{tabular}

\subsubsection{Precision}

The intra- and inter-day precisions of the four tanshinones in QC samples at three concentration levels are presented in Table 3. The intra-day precision (RSD) ranged from $1.41 \%$ to $7.19 \%$ and inter-day precision (RSD) ranged from $3.35 \%$ to $7.80 \%$.

Table 3. The intra- and inter-day precisions of four tanshinones in QC samples at three concentration levels ( $n=6$ replicates per day).

\begin{tabular}{cccccc}
\hline \multirow{2}{*}{$\begin{array}{c}\text { Analytes } \\
\text { Spiked Conc. } \\
\text { (ng/mL) }\end{array}$} & $\begin{array}{c}\text { Measured } \\
\text { Conc. (ng/mL) }\end{array}$ & RSD (\%) & $\begin{array}{c}\text { Measured } \\
\text { Conc. }(\mathbf{n g} / \mathbf{m L})\end{array}$ & RSD (\%) \\
\cline { 3 - 6 } Tanshinone IIA & 4.32 & $4.07 \pm 0.07$ & 1.75 & $4.09 \pm 0.14$ & 3.49 \\
& 21.6 & $19.14 \pm 0.57$ & 3.00 & $19.57 \pm 0.67$ & 3.40 \\
& 108 & $90.75 \pm 1.27$ & 1.41 & $91.95 \pm 4.32$ & 4.70 \\
\hline \multirow{2}{*}{ Cryptotanshinone } & 4.44 & $3.89 \pm 0.23$ & 6.01 & $3.93 \pm 0.31$ & 7.80 \\
& 22.2 & $19.93 \pm 1.17$ & 5.87 & $20.52 \pm 1.17$ & 5.69 \\
Dihydrotanshinone I & 111 & $89.45 \pm 2.03$ & 2.20 & $94.20 \pm 1.15$ & 3.35 \\
\hline \multirow{2}{*}{ Tanshinone I } & 4.56 & $4.23 \pm 0.30$ & 7.19 & $4.22 \pm 0.25$ & 5.93 \\
& 22.8 & $20.82 \pm 0.44$ & 2.12 & $20.91 \pm 1.14$ & 5.46 \\
& 114 & $99.7 \pm 4.37$ & 4.38 & $97.8 \pm 3.72$ & 3.80 \\
\hline
\end{tabular}

\subsubsection{Matrix Effect and Recovery}

The mean matrix effects of the four tanshinones at low, middle, and high concentration ranged from $-8.75 \%$ to $-13.8 \%$ RSD $\leq 8.99 \%$. No obvious matrix effect was observed. The mean extraction recoveries of the four tanshinones in plasma at three different concentration levels were found to be from $84.80 \%$ to $94.51 \%$ with RSD $\leq 7.71 \%$. The data are summarized in Table 4 .

\subsubsection{Stability}

The data of stability experiments are summarized in Table 5, indicating that the four tanshinones in rat plasma were stable after three freeze-thaw cycles and at room temperature for $8 \mathrm{~h}$. 
Table 4. Extraction recovery and matrix effects of four tanshinones in QC samples at three concentration levels $(n=6)$.

\begin{tabular}{cccccc}
\hline Analytes & Spiked Conc. (ng/mL) & Recovery (\%) & RSD (\%) & Matrix Effects (\%) & RSD (\%) \\
\hline \multirow{3}{*}{ Tanshinone IIA } & 4.32 & $93.19 \pm 3.56$ & 3.82 & -13.8 & 8.99 \\
& 21.6 & $90.35 \pm 2.46$ & 2.72 & -10.12 & 5.71 \\
& 108 & $84.80 \pm 3.57$ & 6.45 & -12.83 & 7.89 \\
\hline \multirow{3}{*}{ Cryptotanshinone } & 4.44 & $89.98 \pm 6.93$ & 7.71 & -12.08 & 7.52 \\
& 22.2 & $92.72 \pm 4.67$ & 5.03 & -8.75 & 6.52 \\
Dihydrotanshinone I & 111 & $86.52 \pm 2.79$ & 3.22 & -11.23 & 6.58 \\
\hline \multirow{3}{*}{ Tanshinone I } & 4.56 & $92.17 \pm 3.05$ & 3.30 & -12.8 & 7.46 \\
& 22.8 & $89.51 \pm 5.14$ & 5.74 & 10.88 & 6.34 \\
& 114 & $86.28 \pm 2.79$ & 3.23 & 8.64 & 6.13 \\
\hline
\end{tabular}

Table 5. Room-temperature stability and triple freeze-thaw stability of four tanshinones in QC samples at three concentration levels $(n=6)$.

\begin{tabular}{|c|c|c|c|c|c|}
\hline \multirow[b]{2}{*}{ Analytes } & \multirow{2}{*}{$\begin{array}{l}\text { Spiked Conc. } \\
\text { (ng/mL) }\end{array}$} & \multicolumn{2}{|c|}{ Room-Temperature Stability } & \multicolumn{2}{|c|}{ Freeze-Thaw Stability } \\
\hline & & $\begin{array}{c}\text { Measured } \\
\text { Conc. }(n g / m L)\end{array}$ & RSD (\%) & $\begin{array}{c}\text { Measured } \\
\text { Conc. }(\mathrm{ng} / \mathrm{mL})\end{array}$ & RSD (\%) \\
\hline \multirow{3}{*}{ Tanshinone IIA } & 4.32 & $4.05 \pm 0.24$ & 5.98 & $4.11 \pm 0.24$ & 5.77 \\
\hline & 21.6 & $19.58 \pm 0.57$ & 2.90 & $19.62 \pm 0.37$ & 1.83 \\
\hline & 108 & $90.39 \pm 4.07$ & 4.51 & $92.15 \pm 4.40$ & 4.77 \\
\hline \multirow{3}{*}{ Cryptotanshinone } & 4.44 & $3.91 \pm 0.30$ & 7.56 & $3.86 \pm 0.24$ & 6.20 \\
\hline & 22.2 & $20.47 \pm 0.86$ & 4.21 & $20.27 \pm 1.17$ & 5.75 \\
\hline & 111 & $96.75 \pm 4.09$ & 4.22 & $96.55 \pm 3.31$ & 3.43 \\
\hline \multirow{3}{*}{ Dihydrotanshinone I } & 4.56 & $4.12 \pm 0.18$ & 4.32 & $4.1 \pm 0.16$ & 3.79 \\
\hline & 22.8 & $20.4 \pm 0.88$ & 4.35 & $19.67 \pm 1.48$ & 1.89 \\
\hline & 114 & $96.79 \pm 2.51$ & 2.59 & $94.75 \pm 3.25$ & 3.43 \\
\hline \multirow{3}{*}{ Tanshinone I } & 4.28 & $3.9 \pm 0.24$ & 6.13 & $3.91 \pm 0.24$ & 5.41 \\
\hline & 21.4 & $20.05 \pm 0.89$ & 4.44 & $20.24 \pm 0.89$ & 3.69 \\
\hline & 107 & $92.98 \pm 3.73$ & 4.01 & $91.54 \pm 3.73$ & 2.56 \\
\hline
\end{tabular}

\subsection{Determination of Biochemical Indicators in Adenine-Induced CRF Models}

The analytical results of biochemical indicators were presented in Figure 2. After 14 days of testing, serum creatinine (Scr), blood urea nitrogen (BUN), and urinary protein (UP) in the CRF rats increased significantly compared with control group $(p<0.05$ or $p<0.01$ or $p<0.001)$.
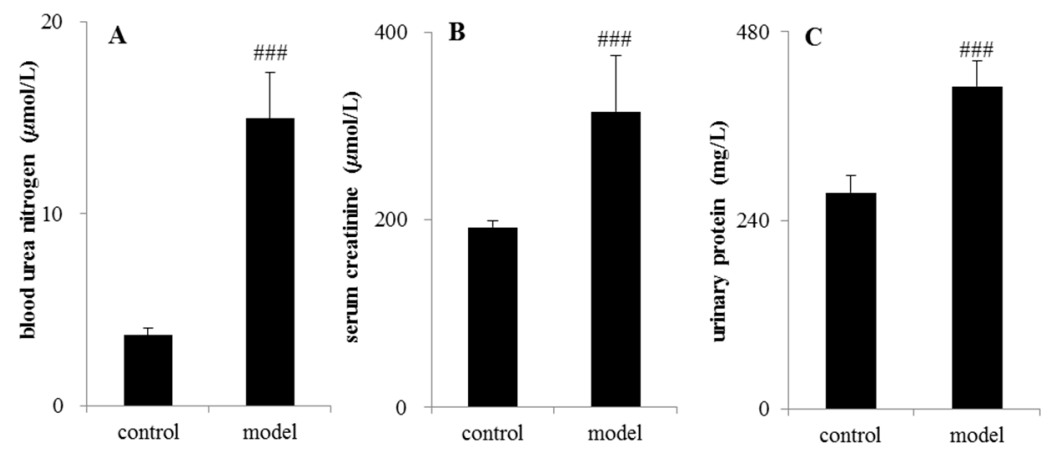

Figure 2. Determination of biochemical indicators between the control group and adenine-induced chronic renal failure group. (A) Blood urea nitrogen; (B) serum creatinine (Scr); (C) urinary protein (UP). ${ }^{\# \#} p<0.001$. 


\subsection{Pharmacokinetics Study}

The validated UPLC-MS/MS method was successfully applied to the pharmacokinetic studies of normal rats administered SMEE or SMEE and SMWE compared with CRF rats $(n=6)$. The profiles of the mean plasma concentration over time for the four tanshinones are illustrated in Figure 3. The main pharmacokinetic parameters calculated by a non-compartment model are presented in Table 6. This was the first report on the pharmacokinetic parameters of four tanshinones in CRF rats and this was also the first report about the influence of SMWE on the pharmacokinetic profile of four tanshinones in CRF rats.

As shown in Table 6, the four tanshinones achieved their maximum plasma concentrations in about $2 \mathrm{~h}$ in M-E (intragastric administration Salvia miltiorrhiza ethanol extract to CRF rats) group and do not show significant difference from C-E (Intragastric administration Salvia miltiorrhiza ethanol extract to normal rats) group except for cryptotanshinone, the $\mathrm{T}_{\text {max }}$ of which was delayed by about $1.5 \mathrm{~h}$ in the $\mathrm{M}-\mathrm{E}$ group. However, the value of $\mathrm{C}_{\max }$ and $\mathrm{AUC}_{0-\mathrm{t}}$ of four tanshinones except for dihydrotanshinone I reduced by $50 \% \sim 80 \%$ and the CLz/F increased by two to four times significantly $(p<0.05)$ in the M-E group, indicating absorption of tanshinone IIA, cryptotanshinone, and tanshinone I were decreased in CRF rats.

Table 6. Pharmacokinetics parameters of the four tanshinones after intragastric administration of SMEE or SMEE and SMWE (mean \pm SD, $n=6$ ). C-E: Intragastric administration Salvia miltiorrhiza ethanol extract to normal rats; M-E: Intragastric administration Salvia miltiorrhiza ethanol extract to CRF rats; C-EW: Intragastric administration Salvia miltiorrhiza ethanol extract and water extract to normal rats; M-EW: Intragastric administration Salvia miltiorrhiza ethanol extract and water extract to CRF rats. ${ }^{*} p<0.05$, ${ }^{* *} p<0.01,{ }^{* * *} p<0.001$ : M-E vs. C-E; ${ }^{\#} p<0.05,{ }^{\# \#} p<0.01,{ }^{\# \#} p<0.001$ : C-EW vs. C-E; ${ }^{+} p<0.05$, ${ }^{++} p<0.01,{ }^{+++} p<0.001$ : M-EW vs. M-E; ${ }^{\Delta} p<0.05,{ }^{\Delta \Delta} p<0.01,{ }^{\Delta \Delta \Delta} p<0.001$ : M-EW vs. C-EW.

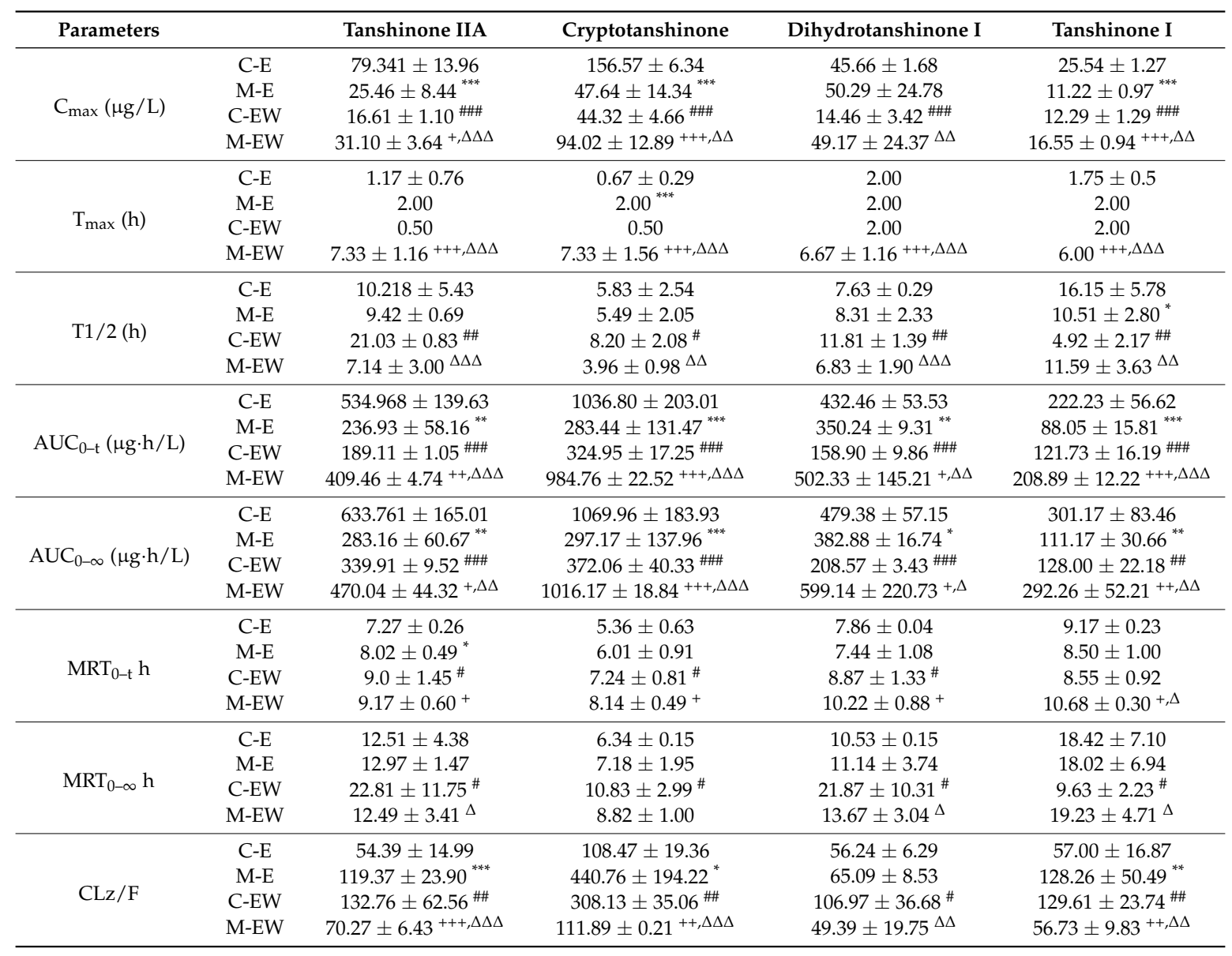


Tanshinone IIA

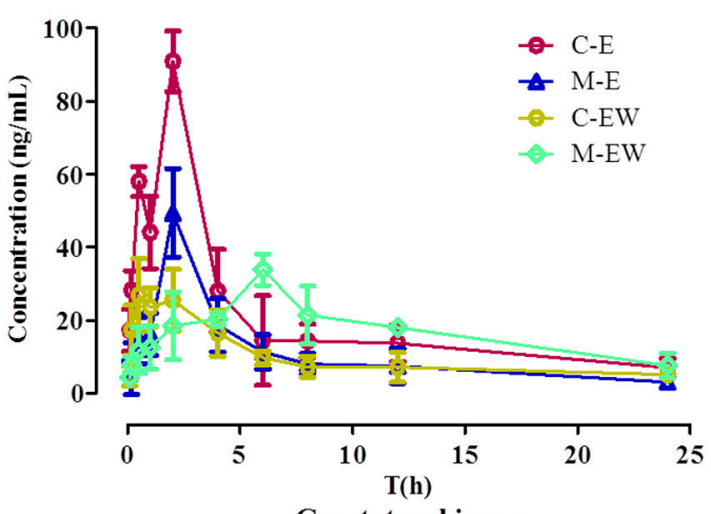

Cryptotanshinone

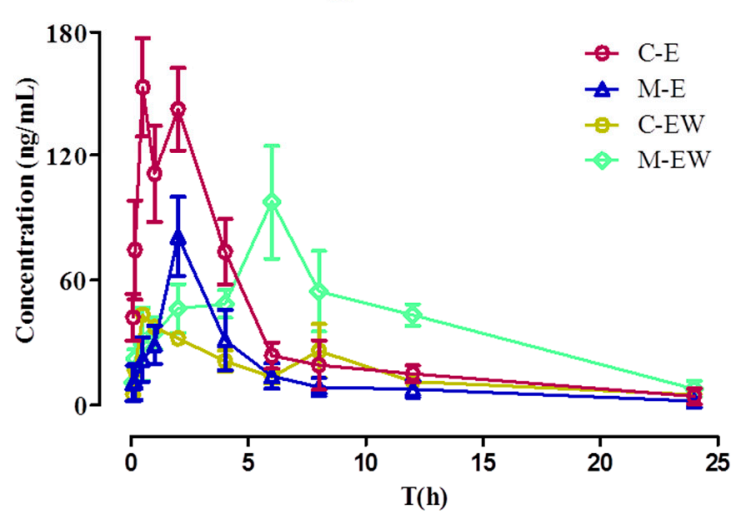

Dihydrotanshinone I

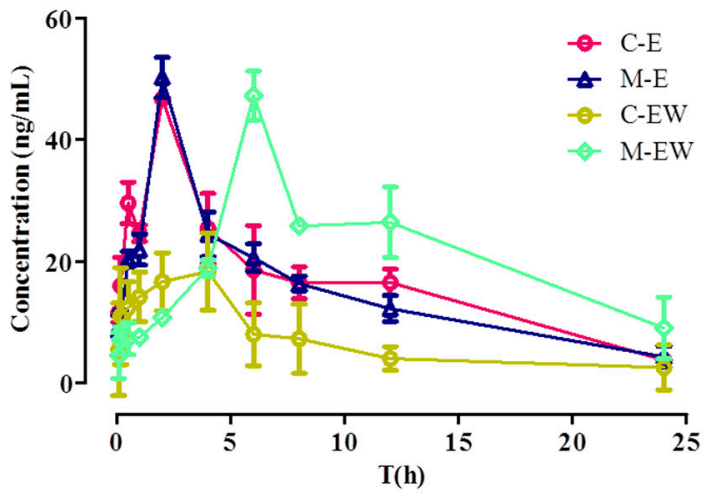

Tanshinone I

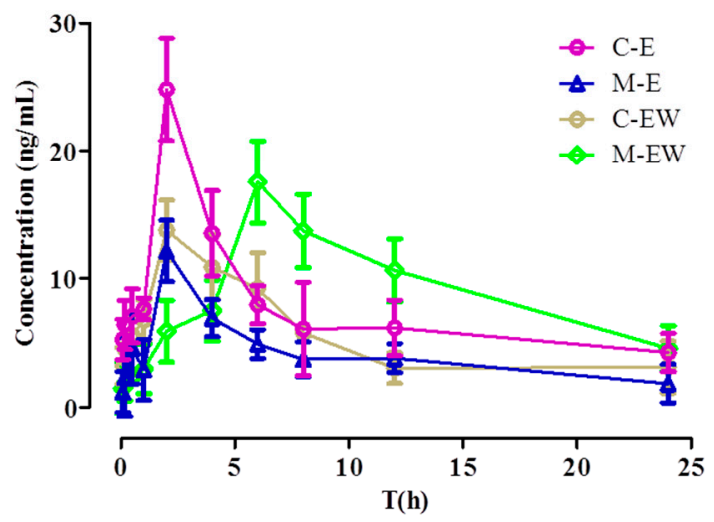

Figure 3. The profiles of the mean plasma concentration versus time of four tanshinones $(n=6)$. C-E: Intragastric administration Salvia miltiorrhiza ethanol extract to normal rats; M-E: Intragastric administration Salvia miltiorrhiza ethanol extract to CRF rats; C-EW: Intragastric administration Salvia miltiorrhiza ethanol extract and water extract to normal rats; M-EW: Intragastric administration Salvia miltiorrhiza ethanol extract and water extract to CRF rats.

After intragastric administration SMWE to C-E group rats, the value of $\mathrm{C}_{\max }$ and $\mathrm{AUC}_{0-\mathrm{t}}$ of tanshinone IIA separately decreased from $79.34 \mu \mathrm{g} / \mathrm{L}$ and $534.97 \mu \mathrm{g} / \mathrm{L}$ to $16.61 \mu \mathrm{g} / \mathrm{L}$ and $189.11 \mu \mathrm{g} / \mathrm{L}$, with CLz/F augmenting from $54.39 \mu \mathrm{g} / \mathrm{L}$ from to $132.76 \mu \mathrm{g} / \mathrm{L}$, and the variation tendency of the other three tanshinones were the same $(p<0.05)$, which was consistent with our previous reports about the pharmacokinetic influences of polyphenolics on tanshinones [31]. Nevertheless, after intragastric administration SMWE to M-E group rats, the value of $C_{\max }, T_{\max }, \mathrm{AUC}_{0-\mathrm{t}}$ of four tanshinones was upregulation and that of $\mathrm{CLz} / \mathrm{F}$ was downregulation, which undulated similarity from the model group to the normal group after intragastric administration with compatibility of SMEE and SMWE. These results hinted that SMWE could improve the bioavailability and absorption of tanshinones in CRF rats, but have a converse effect in normal rats.

Pharmacokinetic processes of drugs in the body are divided into absorption, distribution, metabolism, and excretion. Any changes in the link may cause changes in pharmacokinetic parameters. Previous reports also showed that polyphenolics in Salvia miltiorrhiza can influence the pharmacokinetic of tanshinones in normal rats [28-30], which may be involved in the effect of polyphenolics on blood-brain barrier [32]. Due to the great role of intestinal absorption after intragastric administration and the influence of chronic kidney disease on the structure of gut microbiology [33], the difference of pharmacokinetic parameters of tanshinones in normal and CRF rats may be closely associated with the change of relative enzymes or intestinal bacteria participating in the metabolism, which will be explored by us in the future. 


\section{Materials and Methods}

\subsection{Instruments and Chemicals}

Waters Acquity ${ }^{\mathrm{TM}}$ Ultra Performance LC system (Waters, New York, NY, USA) equipped with a Quattro Micro MS spectrometer and a Waters Xevo ${ }^{\text {TM }}$ G2 TQ MS (Waters MS Technologies, Manchester, UK). MassLynx v4.1 workstation was adopted to analyze the data. Ultrapure water meter (Nanjing Pu Yi Yida Science and Technology Development Co., Ltd., Nanjing, China). Milli-Q system was from Millipore, Bedford, MA, USA. Vacuum freeze-drying equipment (Labconco, UK) and an ultra-high speed centrifuge at low temperature (Thermo, Renfrew, UK) were used. UPLC-grade acetonitrile and formic acid were purchased from Sigma-Aldrich (St. Louis, MO, USA). Standard substance Dihydrotanshinone I (20140516) was bought from Nanjing spring autumn biological engineering Co., Ltd. (Nanjing, China). Tanshinone I (110867-200406), Tanshinone IIA (110766-200619) and Clarithromycin (Internal standard, 130356-200403) were purchased from National Institute for Food and Drug Control (Beijing, China). Cryptotanshinone (110852-200806) were obtained from National Institute for the Control of Pharmaceutical and Biological Products (Beijing, China). Salvia miltiorrhizae Radix et Rhizoma (20150701) was bought from Bozhou city traditional Chinese medicinal materials market.

\subsection{Preparation and Determination of SMEE and SMWE}

The air-dried Salvia miltiorrhizae Radix et Rhizoma (400 g) was extracted with 2 L purified water three times with ultrasound wave extract at ambient temperature. The extraction was concentrated to $1 \mathrm{~g} / \mathrm{mL}$ and precipitated with $80 \%$ ethanol for $24 \mathrm{~h}$ at $4{ }^{\circ} \mathrm{C}$. The supernatant was concentrated and freeze dried into powder as SMWE (62 g). The residue after water extraction was extracted again with 2 L 95\% ethanol three times in the same way. After being concentrated under reduced pressure, it was freeze dried into powder as SMEE (26 g). SMEE (40 mg), and SMWE (50 mg) were separately accurately weighed and dissolved in $10 \mathrm{~mL} \mathrm{90 \%} \mathrm{methanol} \mathrm{containing} \mathrm{IS} \mathrm{with} \mathrm{the} \mathrm{concentration} \mathrm{of}$ $136.56 \mathrm{ng} / \mathrm{mL}$. The solution was centrifuged at 13,000 rpm for $10 \mathrm{~min}$ and $5 \mu \mathrm{L}$ of the supernatant was injected for UPLC-TQ/MS analysis.

\subsection{UPLC-TQ/MS Condition}

The UPLC-TQ/MS condition refers the previous report [31] with some modifications. The quantitative analysis of the chemical constituents was carried out on Thermo Syncronis $\mathrm{C}_{18}$ $(100 \mathrm{~mm} \times 2.1 \mathrm{~mm}, 1.7 \mu \mathrm{m})$ by UPLC-TQ/MS. A gradient mobile phase system was composed by water (containing $0.1 \% \mathrm{HCOOH}$ ) (A)-acetonitrile (B) at a flow rate of $0.4 \mathrm{~mL} \cdot \mathrm{min}^{-1}$. The optimized UPLC elution conditions were: 0 1 min, 3\% 3\% B; 1 6 min, 3\% 30\% B; 6 7 min, 30\% 40\% B; 7 10 min, $40 \% \sim 95 \%$ B, 10 12 $\mathrm{min}, 95 \% \mathrm{~B}, 12 \sim 12.2 \mathrm{~min}, 95 \% \sim 3 \% \mathrm{~B}$. The sample injection was $5 \mu \mathrm{L}$ and the column temperature was maintained at $35^{\circ} \mathrm{C}$. The autosampler was maintained at $4{ }^{\circ} \mathrm{C}$.

The mass spectrometer was operated in the positive multiple-reaction monitoring mode. The capillary and cone voltage were set at $3.0 \mathrm{kV}$ and $30 \mathrm{~V}$, respectively. The desolvation gas was set to $1000 \mathrm{~L} / \mathrm{h}$ at a temperature of $500{ }^{\circ} \mathrm{C}$, the cone gas was set to $50 \mathrm{~L} / \mathrm{h}$ and the source temperature was set to $150{ }^{\circ} \mathrm{C}$. The data acquisition rate was set to $0.2 \mathrm{~s}$. All of the data acquisition and analyses of data were controlled by Waters MassLynx v4.1 software.

\subsection{Standard Solutions Preparation}

The appropriate amounts of four tanshinones were separately weighed and dissolved in methanol as the stock solutions. Mixture stock solution was prepared by mixing and diluting the above-mentioned four stock solutions with methanol to achieve final concentrations of $108 \mu \mathrm{g} / \mathrm{mL}$ for tanshinone IIA, $114 \mu \mathrm{g} / \mathrm{mL}$ for dihydrotanshinone I, $107 \mu \mathrm{g} / \mathrm{mL}$ for tanshinone I, and $111 \mu \mathrm{g} / \mathrm{mL}$ for cryptotanshinone. The mixture stock solution was serially diluted with methanol at ratios of $1: 100,1: 200,1: 1000,1: 2000,1: 10,000,1: 20,000$, and 1:100,000 to provide a series of working 
standard solutions at appropriate concentrations in the range of $0.00108-1.08 \mu \mathrm{g} / \mathrm{mL}$ for tanshinone IIA, $0.00114-1.14 \mu \mathrm{g} / \mathrm{mL}$ for dihydrotanshinone I, $0.00107-1.07 \mu \mathrm{g} / \mathrm{mL}$ for tanshinone I, 0.001 and $11-1.11 \mu \mathrm{g} / \mathrm{mL}$ for cryptotanshinone. These series of working standard solutions after addition IS with the final concentrations of $136.56 \mathrm{ng} / \mathrm{mL}$ drew a curve, which was applied to calculate the content of four tanshinones and five water soluble salvianolic acids in SMEE and SMWE.

The plasma samples at $24 \mathrm{~h}$ from C-E, M-E, C-EW, and M-EW group rats were detected and the concentration were $4.39,3.69,3.58,4.08 \mathrm{ng} / \mathrm{mL}$ for tanshinone IIA; $4.26,4.09,3.81,3.94 \mathrm{ng} / \mathrm{mL}$ for tanshinone I; 4.52, 4.33, 3.88, $4.26 \mathrm{ng} / \mathrm{mL}$ for dihydrotanshinone I; and 4.15, 3.29, 3.82, $4.48 \mathrm{ng} / \mathrm{mL}$ for cryptotanshinone, respectively. The plasma concentration at $24 \mathrm{~h}$ of four tanshinones was close to $4 \mathrm{ng} / \mathrm{mL}$, so the low concentration of quality control (QC) standard work solutions of four tanshinones was set near to $4 \mathrm{ng} / \mathrm{mL}$. Hence, high, middle, and low concentrations of QC standard work solutions were prepared by diluting the highest concentration point of the calibration curve according to the ratios of 1:1, 1:5, and 1:25, respectively. Three concentrations of QC work solutions were 108, 21.6, $4.32 \mathrm{ng} / \mathrm{mL}$ for tanshinone IIA; $114,22.8,4.56 \mathrm{ng} / \mathrm{mL}$ for dihydrotanshinone I; $107,21.4,4.28 \mathrm{ng} / \mathrm{mL}$ for tanshinone I; and 111, 22.2, $4.44 \mathrm{ng} / \mathrm{mL}$ for cryptotanshinone. IS working solution $(136.56 \mathrm{ng} / \mathrm{mL})$ was prepared by diluting the stock solution $(122.5 \mu \mathrm{g} / \mathrm{mL})$ with methanol. All the solutions were stored at $-20^{\circ} \mathrm{C}$.

\subsection{Sample Preparation}

The plasma samples were prepared by one-step direct protein precipitation with three times the volume of methanol.

The calibration curve samples and QC samples were prepared by separately adding $20 \mu \mathrm{L}$ of a series of working standard solutions or $20 \mu \mathrm{L}$ of three concentrations of standard work solutions, to $50 \mu \mathrm{L}$ of blank plasma, then $130 \mu \mathrm{L}$ of IS solution was spiked. Samples of rat plasma (50 $\mu \mathrm{L})$ were mixed with $20 \mu \mathrm{L}$ of methanol and $130 \mu \mathrm{L}$ of IS solution. After vortex-mixing for $30 \mathrm{~s}$ and centrifugation at 13,000 rpm for $10 \mathrm{~min}$ at $4{ }^{\circ} \mathrm{C}, 5 \mu \mathrm{L}$ of the supernatant was injected for UPLC-TQ/MS analysis.

\subsection{Method Validation}

\subsubsection{Specificity}

Blank plasma samples from different rats were prepared and analyzed to eliminate the interferences from endogenous compounds. IS addition to blank sample with analytes was analyzed to investigate the potential interferences of IS. The chromatograms from blank plasma, blank plasma spiked with IS, blank plasma spiked with analytes and IS, as well as plasma samples after oral administration of SMEE were compared.

\subsubsection{Linearity of Calibration Curves and Lower Limits of Quantification (LLOQ)}

The calibration curves for these four compounds were formed by plotting peak-area ratios $(\mathrm{Y})$ of each analyte to the IS versus plasma concentrations $(X)$, using the least-square linear regression with a weighting factor $1 / X$. The ratio of signal to noise above 10 was defined as LLOQ.

\subsubsection{Precision}

The intra-day and inter-day precision of four tanshinones were separately estimated by determining six replicate QC samples at three different concentration (low, medium, and high) levels on the same day and on three consecutive days using calibration curves obtained daily. RSD of the precision must be below $15 \%$ at each concentration level [34].

\subsubsection{Extraction Recovery and Matrix Effect}

The extraction recovery and matrix effect of four tanshinones were determined by analyzing six replicates of plasma samples at three QC concentration levels. The extraction recoveries evaluated by 
comparing the content of the $20 \mu \mathrm{L}$ analytes spiked in $50 \mu \mathrm{L}$ blank plasma precipitated with $130 \mu \mathrm{L}$ IS calculated by standard curve with the actual additive content. The matrix effect was calculated by comparing the peak areas of the $20 \mu \mathrm{L}$ analytes spiked in $50 \mu \mathrm{L}$ blank plasma precipitated with $130 \mu \mathrm{L}$ IS with those spiked in $50 \mu \mathrm{L}$ water addition to $130 \mu \mathrm{L}$ IS.

\subsubsection{Stability}

The stability of all analytes in rat plasma was studied by analyzing six replicate QC samples at three different concentrations including: room-temperature stability, freeze, and thaw stability.

The freeze and thaw stability were determined after three freeze-thaw cycles (from $-80{ }^{\circ} \mathrm{C}$ to $25^{\circ} \mathrm{C}$ ). The room-temperature stability was assessed by analyzing QC samples kept at room temperature for $8 \mathrm{~h}$. All QC samples were determined by using a daily calibration curve. The RSD of stability by calculating the concentration should be within $\pm 15 \%$ [34].

\subsubsection{Animals}

All experimental procedures were carried out in accordance with the Guide for the Care and Use of Laboratory Animals, and before the animal experiments were carried out, the procedures were approved by the Research Ethics Committee of Nanjing University of Chinese Medicine (Nanjing, China). Male SD rats were obtained from the Central Animal Breeding House of Nanjing University of Chinese Medicine (Nanjing, China). They were housed in cages with a constant humidity (ca. $60 \% \pm 2 \%$ ) and temperature (ca. $25 \pm 1{ }^{\circ} \mathrm{C}$ ) with a light/dark cycle of $12 \mathrm{~h}$. The animals were used for six weeks and underwent an adaptation period of several days, during which they were given free access to water and food.

The 24 rats weighing 190 210 g were randomly divided into two groups ( $N=12$ /group), a control group and model group, after measuring their body weight. The model group was given $150 \mathrm{mg} / \mathrm{kg} \cdot \mathrm{d}$ body weight of adenine dissolved with $1 \%(w / v)$ gum acacia solution (by gastric gavage) for 14 days to establish an adenine-induced CRF model. Urine and plasma were collected for biochemical indicator measurements-Scr, BUN, and UP-which were applied to evaluate the model. During those 14 days, the control group was similarly given an equal volume of gum acacia solution every day. Body weight was measured daily for all rats. On the last day, the control rats were divided into C-E group and C-EW group. The model rats were also divided into an $\mathrm{M}-\mathrm{E}$ group and $\mathrm{M}-\mathrm{EW}$ group. Both $\mathrm{C}-\mathrm{E}$ and $\mathrm{M}-\mathrm{E}$ groups were gavaged $0.65 \mathrm{~g} / \mathrm{kg}$ SMEE (equivalent to $16.42 \mathrm{mg} / \mathrm{kg}$ of tanshinone IIA, $13.36 \mathrm{mg} / \mathrm{kg}$ of dihydrotanshinone I, $56.85 \mathrm{mg} / \mathrm{kg}$ of cryptotanshinone, $8.12 \mathrm{mg} / \mathrm{kg}$ of drotanshinone I). Both C-WE and M-WE groups were administered $0.65 \mathrm{~g} / \mathrm{kg}$ of SMEE combined with $1.55 \mathrm{~g} / \mathrm{kg}$ SMWE. Blood samples were collected in $1.5 \mathrm{~mL}$ heparinized tubes via the postorbital venous plexus veins from each rat before drug administration and at $0.083,0.17,0.5,1,2,4,6,8,12$, and $24 \mathrm{~h}$ after drug administration. The blood samples were centrifuged at $3000 \mathrm{rpm}$ for $10 \mathrm{~min}$. The plasma was collected and stored at $-80{ }^{\circ} \mathrm{C}$ until analysis.

\subsubsection{Applications in Pharmacokinetic Studies}

The pharmacokinetic analysis was performed by a non-compartmental approach using the DAS version 3.0 (BioGuider Co., Shanghai, China). The following pharmacokinetic parameters were calculated: area under the plasma concentration-time curve from time zero to infinity ( $\mathrm{AUC}_{0-\infty}$ ), area under the plasma concentration-time curve from zero to the time of last measurable concentration $\left(\mathrm{AUC}_{0-\mathrm{t}}\right)$, maximum observed plasma concentration $\left(\mathrm{C}_{\max }\right)$, time to first occurrence of $\mathrm{C}_{\max }\left(\mathrm{t}_{\max }\right)$, half-life in elimination phase $\left(t_{1 / 2}\right)$, volume of distribution $(\mathrm{Vz} / \mathrm{F})$, mean residence time $(\mathrm{MRT})$.

\section{Conclusions}

In this study, a simple, rapid, and sensitive UPLC-TQ/MS method was successfully established and applied to determine four tanshinones in SMEE before and after combination with SMWE simultaneously in normal and CRF rats. The pharmacokinetic profile of the four tanshinones were 
altered in CRF rats and also varied after oral administration with compatibility of SMEE and SMWE. The results indicated that four tanshinones have slower uptake and higher elimination in CRF rats. After administering the SMWE and SMEE combination, absorption increases and elimination slows down in CRF rats. The results provide scientific information for further exploration of the mechanisms of the combination of SMWE and SMEE and offer a reference for clinical administration of SM.

Acknowledgments: This work was supported by the National Natural Science Foundation of China (Nos. 81373889; 81473408; 81673533) and supported by program for excellent talents in school of pharmacy of Nanjing university of Chinese Medicine (15ZYXET-2). This work was also supported by the Construction Project for Jiangsu Key Laboratory for High Technology Research of TCM Formulae (BM2010576; BK2010561), and a project funded by the Priority Academic Program Development of Jiangsu Higher Education Institutions (ysxk-2014). This work also supported by the 2013 Program for New Century Excellent Talents by the Ministry of Education (Grant NCET-13-0873), 333 High-level Talents Training Project Funded by Jiangsu Province, and Six Talents Project Funded by Jiangsu Province (2012-YY-010).

Author Contributions: The list authors contributed to this work as follows: S.-L.S. and J.D. conceived and designed the experiments, H.-D.C. wrote the paper; H.-D.C. and Y.L. performed the experiment and analyzed the data; Z.Z., S.G., J.G., and Y.Z. guided the experiment; S.-L.S. and D.Q. acquired funding for the research. All authors read and approved the final manuscript.

Conflicts of Interest: All authors declare there is no conflict of interest.

\section{References}

1. Tanonaka, K.; Hirai, K.; Kawaguchi, K.; Ogawa, M.; Yagi, A.; Fujimoto, K. Beneficial effect of Tan-Shen, an extract from the root of salvia, on post-hypoxic recovery of cardiac contractile force. Biochem. Pharmacol. 1990, 40, 1137-1143.

2. Li, Y.H.; Wang, F.Y.; Feng, C.Q.; Yang, X.F. Studies on the active constituents in radix salvia miltiorrhizae and their protective effects on cerebral ischemia reperfusion injury and its mechanism. Pharmacogn. Mag. 2015, 41, 69-73.

3. Cheng, T.O. Danshen, a versatile Chinese herbal drug for the treatment of coronary heart disease. Int. J. Cardiol. 2006, 113, 437-438. [CrossRef] [PubMed]

4. Gao, L.N.; Cui, Y.L.; Wang, Q.S.; Wang, S.X. Amelioration of Danhong injection on the lipopolysaccharidestimulated systemic acute inflammatory reaction via multi-target strategy. J. Ethnopharmacol. 2013, 149, 772-782. [CrossRef] [PubMed]

5. Kang, D.G.; Yun, Y.G.; Ryoo, J.H.; Lee, H.S. Anti-hypertensive effect of water extract of Danshen on renovascular hypertension through inhibition of the renin angiotensin system. Am. J. Chin. Med. 2002, 30, 87-93. [CrossRef] [PubMed]

6. Sun, J.; Huang, S.H.; Tan, B.K.-H.; Whiteman, M.; Zhu, Y.C.; Wu, Y.J.; Ng, Y.; Duan, W.; Zhu, Y.Z. Effects of purified herbal extract of Salvia miltiorrhiza on ischemic rat myocardium after acute myocardial infarction. Life Sci. 2005, 76, 2849-2860. [CrossRef] [PubMed]

7. Zhang, J.H.; Shi, M.Q.; Yang, W.Y.; Liu, G.Y.; Liu, D.D.; Deng, W.; Qin, H.L.; Chen, Z.F. Cardioprotective effects of aqueous extract of Salvia miltiorrhiza on heart failure is related to SDF-1/CXCR4 Axis and Bcl-2 Family Expressions. Adv. Mater. Res. 2014, 998-999, 200-205. [CrossRef]

8. Li, H.Q.; Shi, L.J.; Wei, J.; Zhang, C.P.; Zhou, Z.T. Cellular uptake and anticancer activity of salvianolic acid B phospholipid complex loaded nanoparticles in head and neck cancer and precancer cells. Colloids Surf. B Biointerfaces 2016, 147, 65-72. [CrossRef] [PubMed]

9. Chen, L.J.; Li, Y.; Yin, W.Q.; Shan, W.Q.; Dai, J.F.; Yang, Y.; Li, L. Combination of chlorogenic acid and salvianolic acid B protects against polychlorinated biphenyls-induced oxidative stress through Nrf2. Environ. Toxicol. Pharmacol. 2016, 46, 255-263. [CrossRef] [PubMed]

10. Dong, Z.H.; Ma, D.H.; Gong, Y.; Yu, T.M.; Yao, G. Salvianolic acid B ameliorates CNS autoimmunity by suppressing Th1 responses. Neurosci. Lett. 2016, 619, 92-99. [CrossRef] [PubMed]

11. Chen, B.; Sun, K.; Liu, Y.Y.; Xu, X.S.; Wang, C.S.; Zhao, K.S.; Huang, Q.B.; Han, J.Y. Effect of salvianolic acid B on TNF- $\alpha$ induced cerebral microcirculatory changes in a micro-invasive mouse model. Chin. J. Traumatol. 2016, 19, 85-93. [CrossRef] [PubMed] 
12. Teng, F.K.; Yin, Y.; Cui, Y.J.; Deng, Y.P.; Lie, D.F.; Cho, K.K.; Zhang, G.; Lu, A.P.; Wu, W.Y.; Yang, M.; et al. Salvianolic acid A inhibits endothelial dysfunction and vascular remodeling in spontaneously hypertensive rats. Life Sci. 2016, 144, 86-93. [CrossRef] [PubMed]

13. Ma, S.L.; Zhang, D.W.; Lou, H.X.; Sun, L.R.; Ji, J.B. Evaluation of the anti-inflammatory activities of tanshinones isolated from Salvia miltiorrhiza var. Alba roots in THP-1 macrophages. J. Ethnopharmacol. 2016, 188, 193-199. [CrossRef] [PubMed]

14. Zhang, H.S.; Zhang, F.J.; Li, H.; Liu, Y.; Du, G.Y.; Huang, Y.H. Tanshinone IIA inhibits human esophageal cancer cell growth through miR-122-mediated PKM2 downregulation. Arch. Biochem. Biophys. 2016, 598, 50-56. [CrossRef] [PubMed]

15. Yang, X.; Yan, J.; Feng, J. Treatment with tanshinone IIA suppresses disruption of the blood-brain barrier and reduces expression of adhesion molecules and chemokines in experimental autoimmune encephalomyelitis. Eur. J. Pharmacol. 2016, 771, 18-28. [CrossRef] [PubMed]

16. Tang, H.Y.; He, H.Y.; Ji, H.; Gao, L.L.; Mao, J.W.; Liu, J.; Lin, H.L.; Wu, T.H. Tanshinone IIA ameliorates bleomycin-induced pulmonary fibrosis and inhibits transforming growth factor-beta- $\beta$-dependent epithelial to mesenchymal transition. J. Surg. Res. 2015, 197, 167-175. [CrossRef] [PubMed]

17. Ren, B.; Zhang, Y.X.; Zhou, H.X.; Sun, F.W.; Zhang, Z.F.; Wei, Z.F.; Zhang, C.Y.; Si, D.W. Tanshinone IIA prevents the loss of nigrostriatal dopaminergic neurons by inhibiting NADPH oxidase and iNOS in the MPTP model of Parkinson's disease. J. Neurol. Sci. 2015, 348, 142-152. [CrossRef] [PubMed]

18. Xiang, Q.; Deng, W.; Li, B.; Shi, W.; Wu, J.Y.; Xiang, C.C.; Lv, D.N.; Xie, L.P. Effects of Salvia Miltiorrhiza Extract on Signal Transduction of TSP-1 VEGF and TGF- $\beta 1$ in renal Interstitial Fibrosis Tissues of rats. Guangxi Med. J. 2013, 35, 1582-1585.

19. Shao, M.H.; Wang, C.; Yang, J.; He, L.Q. Effects of Salvianolate on Renal Function and Renal Oxygen Consumption in Rats with Chronic Renal Failure. Acad. J. Shanghai Univ. Tradit. Chin. Med. 2012, 26, 66-69.

20. Wang, Q.L.; Yuan, J.L.; Tao, Y.Y.; Hu, Y.Y.; Liu, C.H. Effect of salvianolic acid B on renal interstitial fibrosis rat induced by $\mathrm{HgCl}_{2}$. Pharmacol. Clin. Chin. Mater. Med. 2008, 24, 12-15.

21. Wang, X.L.; Gao, W.; Sun, M. Pharmacokinetic investigation on interaction between hydrophilic lithospermic acid B and lipophilic tanshinone IIA in rats, an experimental study. J. Tradit. Chin. Med. 2015, 35, 206-210.

22. Wang, J.; Hu, R. Effects of Tanshinone IIA on Renal Nephrin and Transforming Growth Factor- $\beta 1$ of Adriamycin Nephritic Rats. Chin. J. Exp. Tradit. Med. Formula 2011, 17, 245-249.

23. Xu, Y.P.; Huang, K.X.; Pan, Y.; Wang, X.Q.; Yan, P.C.; Ren, Y.P.; Xiang, Z. A rapid UFLC-MS/MS method for simultaneous determination of formononetin, cryptotanshinone, tanshinone IIA and emodin in rat plasma and its application to a pharmacokinetic study of Bu Shen Huo Xue formula. J. Chromatogr. B 2013, 932, 92-99. [CrossRef] [PubMed]

24. Xu, L.Y.; Huang, C.F.; Huang, H.Z.; Li, L.X.; Liang, Y.F.; Guo, B.H.; Li, N. Study on pharmacokinetics of cryptotanshinone and tanshinone IIA from the liposoluble extract of Salvia miltiorrhiza in rats. J. Guangdong Pharm. Univ. 2014, 30, 399-402.

25. Liu, Y.; Li, X.R.; Li, Y.H.; Wang, L.J.; Xue, M. Simultaneous determination of danshensu, rosmarinic acid, cryptotanshinone, tanshinone IIA, tanshinone I and dihydrotanshinone I by liquid chromatographic-mass spectrometry and the application to pharmacokinetics in rats. J. Pharm. Biomed. Anal. 2010, 53, 698-704. [CrossRef] [PubMed]

26. Liu, T.T. Real-Time Quantitative PCR Analysis of Intestinal Lactobacillus Species in Type 2 Diabetic Patients. Ph.D. Thesis, Southern Medical University, Guangzhou, China, 2012.

27. Song, M.; Hang, T.J.; Zhang, Z.X. Comparative study on pharmacokinetics of cryptotanshinone and tanshinones extract of Salvia miltiorrhiza Bge. in rats. Chin. Pharmacol. J. 2008, 43, 51-54.

28. Song, M.; Hang, T.J.; Zhang, Z.X. Comparative study on pharmacokinetics of Pharmacokinetic interactions between the main components in the extracts of Salvia miltiorrhiza Bge. in rat. Acta Pharm. Sin. 2007, 42, 301-307.

29. Song, M.; Hang, T.J.; Zhang, Z.X.; Chen, H.Y. Effects of the coexisting diterpenoid tanshinones on the pharmacokinetics of cryptotanshinone and tanshinone IIA in rat. Eur. J. Pharmacol. Sci. 2007, 32, 247-253. [CrossRef] [PubMed]

30. Ren, H.; Qian, D.W.; Su, S.L.; Guan, H.L.; Zhang, W.; Shang, E.X.; Duan, J.A. Simultaneous determination of tanshinones and polyphenolics in rat plasma by UPLC-TQ/MS and its application to the pharmacokinetic interaction between them. Drug Test Anal. 2016, 8, 744-754. [CrossRef] [PubMed] 
31. Park, E.J.; Ji, H.Y.; Kim, N.J.; Song, W.Y.; Kim, Y.H.; Kim, Y.C.; Sohn, D.H.; Lee, H.S. Simultaneous determination of tanshinone I, dihydrotanshinone I, tanshinone IIA and cryptotanshinone in rat plasma by liquid chromatography-tandem mass spectrometry: Application to a pharmacokinetic study of a standardized fraction of Salvia miltiorrhiza, PF2401-SF. Biomed. Chromatogr. 2008, 22, 548-555. [PubMed]

32. Zhang, X.J. Protect Effect and Mechanism of Salvianolic Acids on the Blood-Brain Barrier Permeability on Mice with Acute Focal Cerebral Ischemia Injury. Ph.D. Thesis, Hebei Medical University, Shijiazhuang, China, 30 March 2015.

33. Xie, S. The Structural Shifts of Gut Micro Biota in Development of Chronic Kidney Disease. Ph.D. Thesis, Southern Medical University, Guangzhou, China, 30 March 2014.

34. Bansal, S.; Arnold, M.; Garofolo, F. International harmonization of bioanalytical guidance. Bioanalysis 2010, 2, 685-687. [CrossRef] [PubMed]

Sample Availability: Samples of ethanol extract and water extract of Danshen and the compounds including tanshinone IIA, cryptotanshinone, dihydrotanshinone I, tanshinone I, danshensu, rosmarinic acid, lithospermic acid, salvianoli acid A, salvianoli acid B are available from the authors.

(C) 2016 by the authors; licensee MDPI, Basel, Switzerland. This article is an open access article distributed under the terms and conditions of the Creative Commons Attribution (CC-BY) license (http://creativecommons.org/licenses/by/4.0/). 